\title{
Chlorofluorocarbon-free inhalers: are we ready for the change?
}

\author{
M.R. Partridge*, A.A. Woodcock+, A.L. Sheffer**, A. Wanner ${ }^{\ddagger}$ A Rubinfeld\#
}

\begin{abstract}
Chlorofluorocarbon-free inhalers: are we ready for the change? M.R. Partridge, A.A. Woodcock, A.L. Sheffer, A. Wanner, A Rubinfeld. CERS Journals Ltd 1998.

ABSTRACT: Chlorofluorocarbons (CFCs) damage stratospheric ozone permitting enhanced levels of ultraviolet B radiation to reach the Earth's surface. As a result, production of CFCs is now banned under the Montreal Protocol with the exception of their temporary continued use in pressurized metered dose inhalers used to treat those with airway disorders. Replacement propellants have now been identified and shown to be safe and a major exercise is under way to reformulate the commonly used aerosolized medicines with the new propellants. The new products are now undergoing clinical trials and the first reformulated $\beta$-agonist and corticosteroid inhalers have reached the marketplace. The majority of the current products will have been changed over to the new types over the next 3 yrs, and each country will adapt a transition strategy to oversee this process.

The politicians, the environmentalists, the pharmaceutical industry and the regulatory authorities have fulfilled their part in this changeover, and respiratory interested health professionals now need to address what this means for them and their patients so that there may be a seamless transition for the millions of people who use inhaled medicines worldwide.
\end{abstract}

Eur Respir J 1998; 11: 1006-1008.
*Chest Clinic, Whipps Cross Hospital, London, UK. +North West Lung Centre, Wythenshawe Hospital, Manchester, UK. **Brigham and Womens Hospital, Boston, MA, USA. Mount Sinai Medical Centre, University of Miami, Florida, USA. \#Royal Melbourne Hospital, Australia.

Correspondence: M.R. Partridge, Chest Clinic, Whipps Cross Hospital, London, E11 1NR, UK

Fax: 441815356709

Keywords: Airway diseases, chlorofluorocarbons, inhalers

The authors are Respiratory Medical Members of the Aerosols, Sterilants, miscellaneous uses and Carbon Tetrachloride Technical Options Committee which makes recommendations to the Technical and Economic Assessment Panel of the United Nations Environment programme. However, the contents of this paper are entirely those of the individuals concerned and do not represent any official policy.
Respiratory physicians are becoming increasingly aware of the adverse environmental effects of groundlevel (trophospheric) ozone on their patients. An adverse effect of ground-level activities on high-level (stratospheric) ozone is about to have a significant impact on our working activities and on our patients. We all need to understand what is happening in the stratosphere, why this has happened, what action governments have taken to remedy the situation, how the pharmaceutical industry has responded to an imminent chloroflurocarbon (CFC) phaseout, and what this will now mean to both physicians and pati-ents.

Ozone in the stratosphere is continually produced and destroyed by physical and chemical processes. It forms a layer $20-40 \mathrm{~km}$ above the Earth's surface, where it absorbs high-energy ultraviolet radiation emitted by the sun, preventing much of this from reaching the surface. In 1974 the Nobel prizewinners M. Molina and S. Rowland postulated that man-made CFCs might damage the ozone layer [1]. These chemicals were widely used in air-conditioning systems, as refrigerants, as foaming agents for plastic packaging, as solvents and as aerosol propellants. Although inert at ground level, as CFCs rise towards the stratosphere the effect of sunlight causes the release of chlorine fragments which destroy ozone. Initially, it was thought that the effect might be small but in 1985 a paper published in Nature by the British Antarctic Survey recorded the first evidence of significant ozone depletion, and these ozone holes have subsequently become larger and deeper and been detected elsewhere [2]. Unfortuna- tely, CFCs take time to reach the stratosphere and, once there, continue to exert their influence for some time, so that even if immediate action is taken to prevent further CFC catalytic damage to stratospheric ozone, it would be followed by a delayed peak of damage before there was a slow recovery in the thickness of the layer. Current predictions are that with the action now being taken, it will take until 2050-2070 to repair the ozone holes.

Thinning of the ozone layer permits increased levels of damaging ultraviolet $B$ (UVB) radiation to reach the Earth's surface. Whilst most of the current increase in skin cancer may be a reflection on human behaviour, increased UVB radiation in the future is likely to lead to a significant increase in all skin cancers including melanomas, as well as enhancing the risk of inducing cataracts and possibly affecting the immune system [3, 4]. Plant life and other ecosystems are also likely to suffer damage [5].

As a result of this scientific understanding, global government action was clearly needed and 10 yrs ago historic international collaboration to protect the environment resulted in the Montreal Protocol. Signatory countries committed themselves to phasing out of CFCs and, after a series of amendments and acceleration of dates, it was determined that this would be by 1996 in developed countries and by 2010 in developing (Article 5) countries [6]. Almost imperceptibly, aerosol deodorants, furniture polishes and hair sprays were reformulated with alternative, often inflammable propellants, and CFCs were phased out as refrigerants and in air-conditioning plants. In anticipation of the phase-out, many of the major pharmaceutical companies 
collaborated as an International Pharmaceutical Aerosol Consortium to look for alternative propellants and to undertake joint toxicological testing of suitable substances. Two such propellants, hydrofluoroalkanes (HFA)-134a and HFA-227, have been shown to be safe, to have no ozonedamaging potential and to have much less global-warming effect than existing CFC propellants (most work having been done so far on HFA-134a). It has been estimated that the cost of the safety assessment programme for this propellant alone was $>$ US\$ 20 million. Now that the propellants are considered to be safe, individual companies must undertake the considerable task of reformulating each of their products with one or other of the new aerosol propellants. The size of this task should not be underestimated and has necessitated major revision of the canisters, valves and elastomers, followed by extensive testing of the combination of new propellants and active medication, with different clinical trials being required by different regulatory authorities.

Given the size of the task it was not surprising that it has taken some time and it was clear that new products would not be available by the phase-out date of 1996 (1995 in the European Union). The Montreal Protocol allowed for this eventuality by means of an essential-use exemption. "Essentiality" is defined as when: 1) there are no technically feasible alternatives to the use of a CFC in the product; 2) the product provides a substantial health environmental or other public benefit that would not be obtainable without the use of the CFC; and 3) the use does not involve a significant release of CFCs into the atmosphere or, if it does, the release is warranted by the consequence if the use were not permitted. An application for essential-use exemption for the continued use of halons (which also have ozone-damaging potential) in fire-fighting systems was rejected. Similarly, the use of CFC propellants in metered dose steroid nasal pumps was deemed to be non-essential. The use of CFCs in metered dose inhalers (MDI) for the treatment of asthma and chronic obstructive pulmonary disease was thought to fulfil the criteria of essentiality. Essentiality is re-examined on a biannual basis and the signatories to the Montreal Protocol are advised by a preparatory meeting of a body known as the Open Ended Working Group (OEWG). The OEWG is advised by three advisory panels, one of which is the Technical and Economic Assessment Panel (TEAP) which coordinates the work of seven Technical Options committees (TOCs), one of which looks specifically and in depth at aerosol use. Each TOC contains a mixture of technical experts and clinicians who do not make policy but make assessments, identify alternatives, highlight their advantages and disadvantages, answer queries and make recommendations to the TEAP. The parties have now asked the TEAP (advised by the TOC) to provide a report on suitable transition strategies to be adopted to cover the phase-out of CFC-containing MDI as the reformulated HFA propellant products become available.

Draft strategies have already been proposed by the Food \& Drugs Administration (FDA) in the USA, by government departments in the UK and Australia, and by the European Community. Any strategy will have as its prime aim the safety of patients and several methods are possible. Transition could occur brand by brand, drug by drug or category by category and could be driven at different rates in different countries, with the ultimate incentive for change being the withdrawal of essential-use exemption by the parties.

In brand-by-brand transition each company reformulating a branded product with nonCFC propellants would phase out the old CFC-containing product over a reasonable, negotiated period. (By itself, this form of policy would have no effect on those generic CFC-containing products that are not being reformulated.) In drug-by-drug transition, it could be deemed that once a certain drug is available in a reformulated form, and has undergone a reasonable period of postmarketing surveillance then, irrespective of manufacturer, all other CFC-containing versions of that drug are withdrawn. This type of transition could be varied to permit more than one (two or three) companies to reformulate before phase-out and the period for phase-out could also vary. This allows consumer choice yet encourages phase-out of CFC-containing MDI.

Category-by-category transition is similar to drug-bydrug transition, but would, for example, look at all $\beta$-agonists as a category and all inhaled glucocorticosteroids as a category. Thus, when considering CFC-containing glucocorticosteroid inhalers, for example, it could be proposed that once one, two or three types of reformulated glucocorticosteroid of any type were available, all CFC-containing inhaled steroids would be phased out. Again, this possible form of transition could be fine tuned to allow more than one company to reformulate each glucocorticosteroid, or be varied to permit no transition to occur until all types of inhaled glucocorticosteroids were available.

Clearly, whichever form of transition strategy is adopted or recommended nationally or internationally, patient safety is paramount and decisions and time-scales must accommodate the need for adequate time for both patient and health professional education, and for adequate postmarketing safety surveillance of the new products.

The transition has already started and the type of transition strategy recommended is likely to vary from one part of the world to another, but versions of both $\beta$-agonist bronchodilators and inhaled glucocorticosteroids have now been reformulated, and it is likely that the majority of CFC-containing MDI will have been reformulated with the new propellants within the next 3 yrs. We need to be aware of what this might involve in terms of cost, workload and preparation of patients to ensure that transition is, wherever possible, seamless.

Pricing policies vary from country to country but it is likely that branded HFA-containing MDI will be introduced at a cost comparable to the branded CFC product they replace. In many countries, the last few years have seen a change from the use of branded products to the use of cheaper generic inhalers. However, many of the manufacturers of the generic inhalers have had neither the research base nor the resources to reformulate their products, and it is therefore likely that whichever transition strategy is adopted, the process will involve a switch from generic to branded products with associated increased costs. Costs may also rise if transition involves a switch from one drug within a category, for example, inhaled glucocorticosteroids, to another, more expensive glucocorticosteroid. This may occur if reformulation proceeds at a different pace for different drugs owing to differences in the ease of the reformulation process. Finally, health providers, governments and individuals need to realise that if 
transition involves a switch not from CFC-containing MDI to HFA-containing MDI but to dry powder devices (which is a reasonable option for many patients and many drugs), this is likely to increase costs.

Not enough is known about many of the reformulated products to know what their introduction will involve for the patient or doctor. For those products about which information is available, it is clear that reformulation often results in changes to the shape and feel of the inhaler, oropharyngeal deposition of aerosol, changes in taste and, in some circumstances, changes in pulmonary deposition of aerosolized drugs which may necessitate dose changes.

Patients and their families need to be informed of the reasons behind this change and reassured that the previous propellants were environmentally damaging and not damaging to the individual. They need to be reassured regarding the safety and efficacy of the new products, and all of these messages need to be given in a consistent manner by all members of the health professional team, including doctors, nurses, pharmacists, respiratory therapists and physiotherapists. These spoken messages then need reinforcement by the use of the written word and other audiovisual methods. Individual physicians, health cooperatives, health providers and hospitals will have to ascertain individually the best method for changing over their patients. This may involve letters to patients forewarning them that the next prescription will be changed and outlining the key messages, or it may involve extra consultations, and these will be essential when transition involves a dosage change or a switch to a dry powder device. Transition is likely to be less problematical if it is undertaken in a co-ordinated way, and having a district or locality policy for changeover may reduce problems for patients moving between primary and secondary care, and for pharmacists by reducing the quantity of dual stocks they maintain. At every stage the patient needs reassurance as to the safety and efficacy of the new products but to be advised of the differences that they may note with the new inhaler.

This story started with a hypothesis. The hypothesis was proven and international government action was initiated. The pharmaceutical industry responded to a threat to the most widely used and recommended method of treating airway disorders, the metered dose inhaler. The regulatory authorities are now licensing the new products and we, as physicians, need to be ready to help our patients to change to using them in a logical and organized way.

\section{References}

1. Molina MJ, Rowland FS. Stratospheric sink for chlorofluoromethanes: chlorine-atom catalysed destruction of ozone. Nature 1974; 249: 810-814.

2. Farman JC, Gardiner BG, Shanklin JD. Large losses of total ozone in Antartica reveal seasonal CLOx/NOx/C interaction. Nature 1985; 315: 207-210.

3. Lloyd SA. Stratospheric ozone depletion. Lancet 1993; 342: 1156-1158.

4. Jeevan A, Kripke ML. Ozone depletion and the immune system. Lancet 1993; 342: 1159-1160.

5. Young AR. The biological effects of ozone depletion. $\mathrm{Br}$ J Clin Pract (Suppl) 1997; 89: 10-15.

6. United Nations Environment Programme Technology and Economic Assessment Panel of the Montreal Protocol on substances that deplete the ozone layer. Part III Update of the 1994 T.O.C. Aerosols Report and the M.D.I. Transition Strategy, TEAP 1996. 